\title{
Epidemiology of ruptured brain arteriovenous malformation: a National Cohort Study in Korea
}

\author{
Tackeun Kim, MD,,2 O-Ki Kwon, MD, PhD,,2 Jae Seung Bang, MD,,2 Heeyoung Lee, MD, PhD, ${ }^{3}$ \\ Jeong Eun Kim, MD, PhD, ${ }^{2,4}$ Hyun-Seung Kang, MD, PhD, ${ }^{2,4}$ Won-Sang Cho, MD, PhD, ${ }^{2,4}$ and \\ Chang Wan Oh, MD, PhD ${ }^{1,2}$
}

1Department of Neurosurgery, Seoul National University Bundang Hospital, Seongnam; ${ }^{2}$ Department of Neurosurgery, Seoul National University College of Medicine, Seoul; ${ }^{3}$ Center for Preventive Medicine and Public Health, Seoul National University Bundang Hospital, Seongnam; and ${ }^{4}$ Department of Neurosurgery, Seoul National University Hospital, Seoul, Republic of Korea

\begin{abstract}
OBJECTIVE Brain arteriovenous malformation (BAVM) is a rare cerebrovascular disease that causes intracranial hemorrhage. Although several reports have demonstrated the epidemiological features of BAVM in Western countries, no epidemiological investigations regarding BAVM have been reported in Korea. The authors aimed to investigate the national epidemiology of ruptured BAVM in a Korean population.
\end{abstract}

METHODS The authors used data from the National Health Insurance Service-National Sample Cohort (NHIS-NSC). The original cohort included approximately 1 million individuals ( $2 \%$ of the Korean population) with 12-year claim data (2002-2013). To construct an initial cohort for investigation, the authors selected 1,016,820 registered individuals in 2005. Subjects with a history of cerebrovascular disease (code I6xx) and BAVM (Q282) between 2002 and 2004 were washedout to identify incident cases. During the 9-year follow-up (2005-2013), the incidence of BAVM was calculated using the earliest date of diagnosis of ruptured or unruptured BAVM. Direct standardization was applied to the crude incidence. Mortality and disability were evaluated using registration data. Related diagnostic procedures were also analyzed.

RESULTS A total of 8,802,696 person-years of observation were noted. During observation, 308 patients were diagnosed with a ruptured BAVM. The crude incidence of ruptured BAVM was 3.5 per 100,000 person-years. There was no sex difference in incidence. The mortality rate for patients with a ruptured BAVM 1 month after diagnosis was $12.7 \%$. At 1-year and 5-year follow-up examinations, mortality rates were $17.2 \%$ and $22.1 \%$, respectively. Severe disability-free survival rates of patients with ruptured AVMs were $75.3 \%$ and $69.8 \%$ at 1-year and 5-year follow-up, respectively.

CONCLUSIONS The standardized incidence of ruptured BAVMs was 3.6 per 100,000 person-years in Korea. Ruptured BAVMs resulted in high mortality and disability rates.

https://thejns.org/doi/abs/10.3171/2018.1.JNS172766

KEYWORDS arteriovenous malformation; brain arteriovenous malformation; cohort; epidemiology; incidence; hemorrhage; nationwide; vascular disorders

$\mathrm{B}$ RAIN arteriovenous malformation (BAVM) is a rare cerebrovascular disease. Valuable investigations in Western societies have revealed that the incidence of BAVM is $0.89-2.05$ per 100,000 person-years. ${ }^{2,47,20}$ BAVM can result in hemorrhage due to high-flow and lowresistance shunting. Hemorrhage resulting from ruptured BAVM (RBAVM) accounts for $4 \%$ of all intracranial hemorrhages. ${ }^{3}$ However, the proportion of BAVM hemorrhage is considerably higher (approximately 30\%) among nontraumatic intracranial hemorrhages in young adults. ${ }^{21}$ The mortality rate of patients with RBAVM has been reported as $4 \%-29 \%{ }^{5,6,9,22}$ Furthermore, the rate of significant disability after hemorrhage is high (23\%-85\%). Even unruptured BAVM (UBAVM) can cause delayed hemorrhage. Therefore, BAVM represents a serious clinical problem with significant negative effects on the quality of life.

To our knowledge, no epidemiological investigations regarding RBAVM involving a Korean cohort have been reported. It is known that certain cerebrovascular diseases have different epidemiological features in Western and

ABBREVIATIONS BAVM = brain arteriovenous malformation; DSA = digital subtraction angiography; NHID = National Health Information Database; NHIS-NSC = National Health Insurance Service-National Sample Cohort; RBAVM = ruptured BAVM; UBAVM = unruptured BAVM.

SUBMITTED November 1, 2017. ACCEPTED January 16, 2018.

INCLUDE WHEN CITING Published online June 29, 2018; DOI: 10.3171/2018.1.JNS172766. 
Eastern countries..$^{14,15}$ Therefore, it would be valuable to investigate the epidemiology of RBAVM using Korea as a representative Asian country. Considering the rare incidence rates reported in previous articles, estimating the national epidemiological features of this disease requires a large sample size. Therefore, this study was designed as a retrospective cohort study using the National Health Insurance Service-National Sample Cohort (NHIS-NSC), which contains detailed information relating to medical utilization by approximately 1 million subjects during a period of 12 years. The aim of this study was to establish the epidemiological features of RBAVM in a Korean population, using retrospective sampling.

\section{Methods \\ NHIS-NSC}

Since 1963, the NHIS has provided for $97 \%$ of the people in Korea. The Medical Aid program provides for the remaining 3\%. All claims have been recorded in a centralized database (National Health Information Database [NHID]), with accompanying information regarding diagnostic codes, types of procedures, drug prescriptions, and related costs. In terms of diagnostic codes, the NHIS uses the Korean Classification of Diseases system, which is based on the International Classification of Diseases, Tenth Edition (ICD-10). Information regarding death and disability is also recorded. From the target population $(46,605,433$ individuals in the 2002 NHID), a representative sample cohort of approximately 1 million participants was randomly selected. Systematic stratified random sampling with proportional allocation within 1476 strata was conducted. Strata were defined by 18 age groups (infants under 1 year, ages 1-4, 5-year age groups between 5 and 79 , and 80 years and above), two groups according to sex (male, female), and 41 groups based on participant's income level. This NHIS-NSC comprised approximately $2.2 \%$ of the Korean population. The NHIS-NSC is openly available for research purposes depending on review board approval. ${ }^{17}$ To mask personal information, alternative identification numbers were used instead of the actual Korean registry identification numbers. Some sensitive information, such as that related to pregnancy, addiction, and sexual abuse, was eliminated from the database. The observation period of the entire NHIS-NSC was 2002-2013.

\section{Cohort Design}

Originally, the NHIS-HSC featured a dynamic cohort: a newborn baby was recruited to replace a deceased individual to maintain the cohort size. However, we decided to remove deceased subjects and did not include newly registered newborn babies after initial registration. To secure a 3-year washout period, we used 2005 as the index year. Subjects with any history of cerebrovascular disease (code I6xx) including RBAVM (I608) between 2002 and 2004 were excluded from the initial cohort. ${ }^{14}$ The age distribution of the final cohort was similar to that of the national population, as determined by the 2005 population and housing census in Korea (Fig. 1). The total observation period of the reconstructed cohort was 9 years (2005-2013).

\section{Data Extraction}

Demographic data, such as those relating to sex and age, were obtained from the database. We classified age according to decade. Subjects with RBAVM were defined as follows: 1) subjects with RBAVM (I608) or BAVM (Q282) diagnostic codes with any simultaneous hemorrhagic stroke (I60x, I61x, and I62x); 2) subjects with hospitalization expense claims at the time of initial diagnosis; and 3) subjects with claims for diagnostic modalities such as CT, MRI, or digital subtraction angiography (DSA) within 2 weeks from the initial diagnosis.

The primary endpoint was the earliest date of registration of a diagnostic code indicating RBAVM or BAVM with any hemorrhagic stroke. The secondary endpoints were the date of registration of a disability code and the date of death. Disability grading in Korea is performed according to a 6-level system (1 for severest disability and 6 for mildest). In the NHIS-NSC, disability grades are dichotomized into 2 groups: mild disability (Grades 3-6) and severe disability (Grades $1-2$ ).

Data relating to the use of diagnostic modalities were extracted using electronic data interchange codes: CT with contrast agent (HA461), brain CT angiography (HA471), brain MRI with or without MR angiography (HE101, HE201, HE501, HE135, HE235, and HE535), and DSA (HA601-HA606). Due to diversity of codes related to treatment and the uncertainty of the relationship with RBAVM, analysis of the treatment of RBAVM was not considered. Initial neurological status could not be assessed neither, because claim data did not include medical records.

\section{Statistical Analysis}

We performed data manipulation and extraction using the open source relational database management system MariaDB (version 10.1.17; MariaDB foundation; http:// mariadb.org). Statistical analysis was performed using $\mathrm{R}$ (version 3.3.1; the R Foundation for Statistical Computing; http://www.r-project.org) and its packages (survival; http:// cran.r-project.org/package=survival).

The crude incidence of RBAVM was directly calculated based on the cohort's observation data using the follow formula:

Crude incidence $=$ number of events/total observation period $\times 100,000$.

Direct standardization of the incidence was achieved by applying the proportions of sex and age groups from the census data of 2005 in Korea to adjust for demographic bias within the cohort as follows: $\Sigma_{\mathrm{i}} \mathrm{P}_{\mathrm{i}} \times \mathrm{I}_{\mathrm{i}}$, where $P_{i}$ is the proportion of each sex and age group from the 2005 census and $I_{i}$ is the incidence of each sex and age group of the current cohort. Sex difference in incidence was tested using a conditional maximum likelihood estimate of rate ratio. Mortality and disability rates were analyzed using Kaplan-Meier survival analysis for each duration.

\section{Results \\ Incidence of RBAVM}

A total of 1,016,820 subjects were registered in 2005. A total 18,652 subjects with a history of cerebrovascu- 


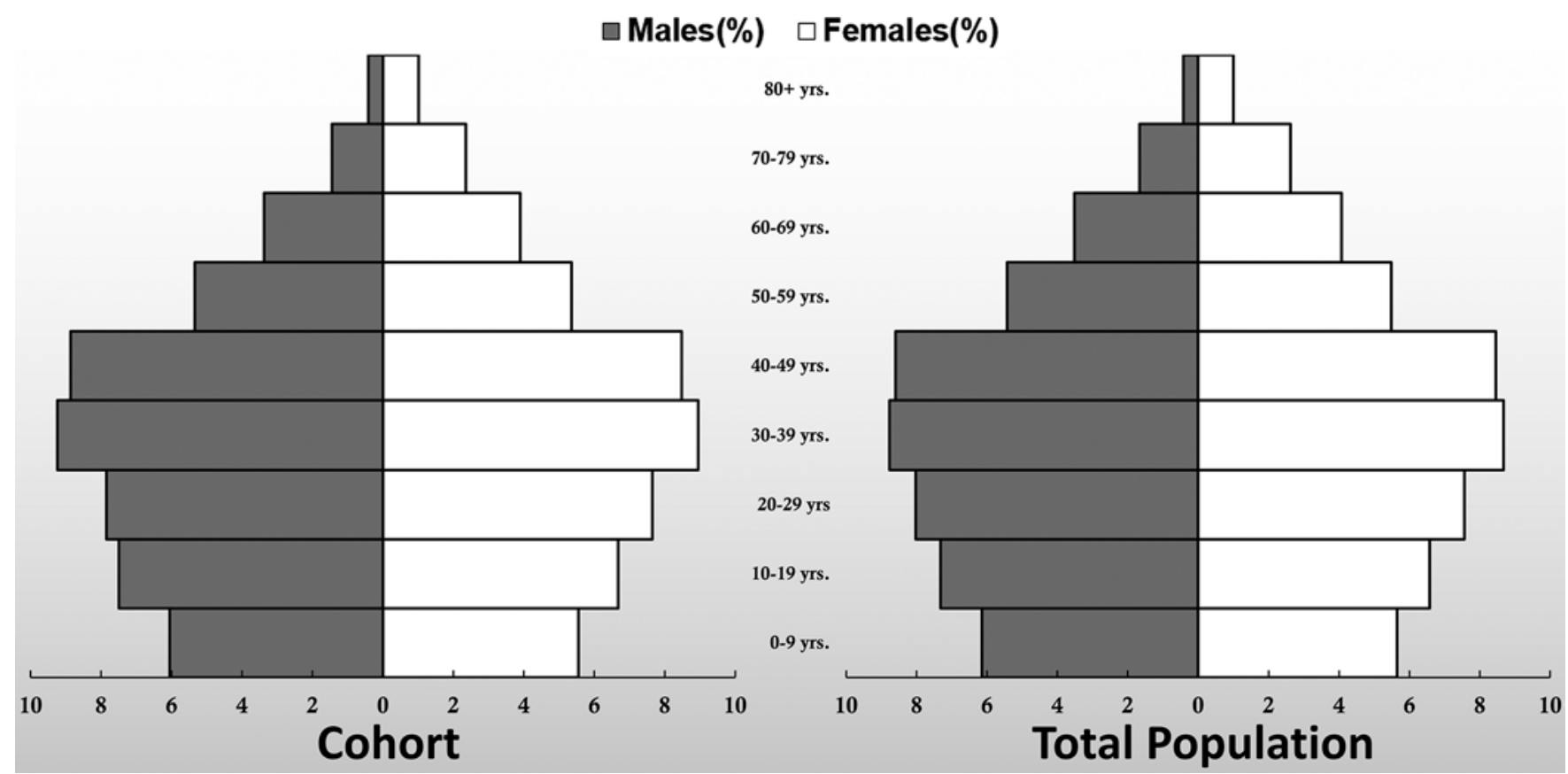

FIG. 1. Pyramid charts of the population structures. The population structure of the cohort shows almost the same distribution as the national population according to the 2005 population and housing census in Korea.

lar disease (I6xx) and BAVM (Q282) between 2002 and 2004 were washed-out to identify incident cases. During the observation period, 39,786 subjects died, including 65 who had been diagnosed with RBAVM. By summing each survival duration, a total of 8,802,696 person-years of observation was established. During observation, 308 subjects were diagnosed with RBAVM (Fig. 2). The majority of RBAVM patients were identified through code I608 $(\mathrm{n}=285,92.5 \%)$, regardless of other hemorrhage code. Twenty-three patients (7.5\%) were detected by combination of BAVM (Q282) and any intracranial hemorrhage (I60x, I61x, and I62x). Accordingly, the crude incidence of RBAVM in Korea was measured as 3.5 per 100,000 person-years (95\% CI 3.1-3.9). The sex- and age-standardized incidence of RBAVM was 3.6 per 100,000 person-years.

Among the 308 patients, there were 144 males (46.8\%) and 164 females (53.2\%). As the observation periods were $4,397,973$ and 4,404,723 person-years in males and females, crude incidences were 3.3 (95\% CI 2.8-3.9) and 3.7 (95\% CI 3.2-4.3) per 100,000 person-years, respectively. The male-to-female incidence ratio was $0.9(95 \%$ CI 0.7-1.1), which indicated no significant sex difference in incidence. Male-to-female ratios of incidence by age groups were not statistically significant throughout cohort (Table 1).

Most RBAVM subjects belonged to the 40- to 49-yearold age group $(\mathrm{n}=78 ; 25.3 \%)$, with the next most in the 50- to 59-year-old age group $(\mathrm{n}=59 ; 19.2 \%), 60$ - to 69 -year-old age group $(n=54 ; 17.5 \%)$, and 30 - to 39 -yearold age group $(n=45 ; 14.6 \%)$. The incidence of RBAVMs in the older age group was higher than that in the younger age groups (Fig. 3).

As a diagnostic modality, DSA was performed in 135 patients (43.8\%). MRI and enhanced CT scans were used for diagnosis in $172(55.8 \%)$ and 51 (16.6\%) patients, respectively (allowing duplicates).

\section{Outcomes of RBAVM}

Among 308 RBAVM patients, 65 (21.1\%) died during a mean follow-up period of $9 \pm 18$ months. Most deaths occurred within 1 month after diagnosis $(n=39 ; 60.0 \%)$. Fifty-two patients $(80.0 \%)$ died within 1 year. The overall survival rate of RBAVM patients within 1 month after diagnosis was $87.3 \% \pm 1.9 \%$. During the 1-year and 5-year

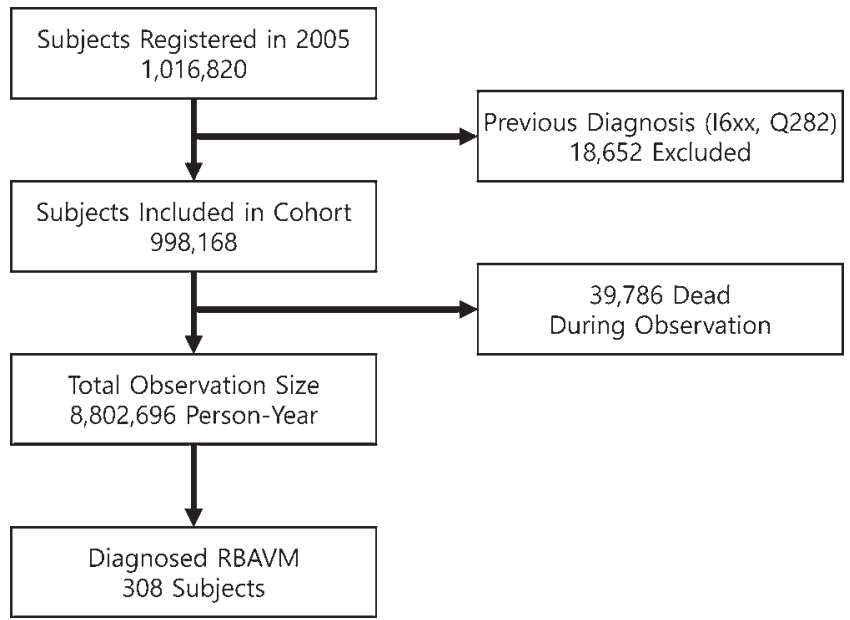

FIG. 2. Flowchart of the number of subjects during each stage of the investigation. 
TABLE 1. Incidence and ratio of RBAVMs by sex and age

\begin{tabular}{|c|c|c|c|c|c|c|c|c|}
\hline \multirow{2}{*}{$\begin{array}{c}\text { Age Group, } \\
\text { yrs }\end{array}$} & \multicolumn{2}{|c|}{ Observation Size* } & \multicolumn{2}{|c|}{ Incident Cases } & \multicolumn{4}{|c|}{ Incidence $†$} \\
\hline & Male & Female & Male & Female & Male & Female & Male/Female Ratioł & $95 \% \mathrm{Cl} \ddagger$ \\
\hline $0-9$ & 543,527 & 497,998 & 5 & 1 & 0.9 & 0.2 & 4.6 & $0.6-109.0$ \\
\hline $10-19$ & 672,067 & 599,382 & 11 & 8 & 1.6 & 1.3 & 1.2 & $0.5-3.2$ \\
\hline $20-29$ & 703,123 & 686,057 & 10 & 6 & 1.4 & 0.9 & 1.6 & $0.6-4.8$ \\
\hline $30-39$ & 825,620 & 801,896 & 20 & 25 & 2.4 & 3.1 & 0.8 & $0.4-1.4$ \\
\hline $40-49$ & 784,032 & 757,794 & 33 & 45 & 4.2 & 5.9 & 0.7 & $0.4-1.1$ \\
\hline $50-59$ & 463,832 & 475,468 & 31 & 28 & 6.7 & 5.9 & 1.1 & $0.7-2.0$ \\
\hline $60-69$ & 278,933 & 338,956 & 24 & 30 & 8.6 & 8.9 & 1.0 & $0.6-1.7$ \\
\hline $70-79$ & 105,700 & 188,051 & 8 & 14 & 7.6 & 7.4 & 1.0 & $0.4-2.4$ \\
\hline $80+$ & 21,138 & 59,121 & 2 & 7 & 9.5 & 11.8 & 0.8 & $0.1-3.6$ \\
\hline Sum & $4,397,973$ & $4,404,723$ & 144 & 164 & 3.3 & 3.7 & 0.9 & $0.7-1.1$ \\
\hline
\end{tabular}

* Observation size is presented in person-years.

$\dagger$ Incidence is presented per 100,000 person-years.

$\ddagger$ Incidence ratio and $95 \% \mathrm{Cl}$ were calculated by conditional maximum likelihood estimate of rate ratio.

follow-up periods, the survival rates were $82.8 \% \pm 2.2 \%$ and $77.9 \% \pm 2.6 \%$. The severe disability-free survival rate was $75.3 \% \pm 2.5 \%$ at the 1-year follow-up after diagnosis. It decreased to $69.8 \% \pm 2.9 \%$ at the 5-year follow-up.

\section{Discussion}

We calculated an estimated crude incidence of RBAVM of 3.5 per 100,000 person-years (95\% CI 3.1-3.9) in Korea using the NHIS-NSC. More precisely, this rate reflected the detection of RBAVMs rather than incidence. However, RBAVM usually causes severe symptoms, including neurological deterioration, and the proportion of potentially undetected RBAVMs would be rare. Moreover, due to the relatively low incidence of BAVM, it could be negligible because the number of undetected BAVM patients divided by the large number of subjects in the cohort was close to 0 . Therefore, we used the term "incidence" in this study rather than "detection rate."

Although this was a cohort investigation, through direct standardization using the national population structure, we tried to secure external validity. The standardized incidence of RBAVM was 3.6 per 100,000 personyears, which was somewhat higher than the crude value. This value is higher than those reported by all previous population-based studies of BAVM. . $^{1,10,11,13,20}$ According

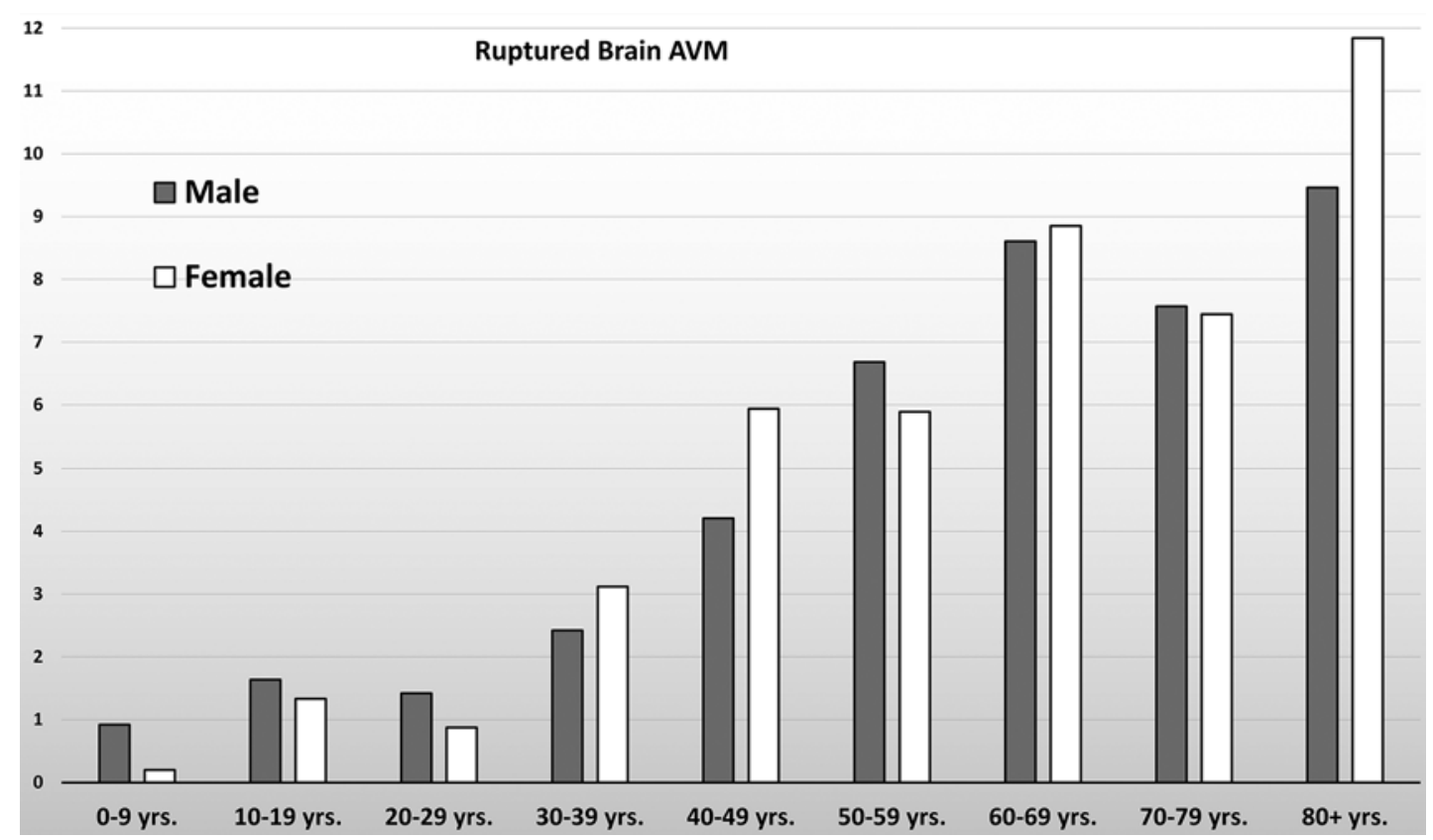

FIG. 3. Incidence of RBAVM according to age and sex. The incidence is higher for older age groups. 
to Gabriel et al., the incidence of BAVM was $1.3(95 \%$ CI 1.2-1.4) in a meta-analysis of 6 studies. ${ }^{10}$ These discrepancies in incidence could be explained by a number of factors. The properties of data sources are important factors. Compared with previous studies, we used a claim database provided by universal insurers operated by the government that is required by law to report all medical expenses by healthcare institutes in Korea. Therefore, the detection rate of rare diseases such as RBAVM could be higher than that of previous studies. To avoid overdetection, we tried to determine BAVM subjects according to the following considerations. First, NHIS claim data were reviewed by the Health Insurance Review and Assessment service. NHIS provides financial support for severe illness including intracranial hemorrhage. Patients with vascular malformations presenting with intracranial hemorrhage can be also supported by NHIS. Thus, a diagnosis of RBAVM is not beneficial over other vascular malformations, including cavernous malformations. Because of the financial load of NHIS, payments from the insurer to the healthcare facilities were strictly controlled by checking the relevance of the diagnostic code related with any intracranial hemorrhage. Second, the diagnostic codes for BAVM tended to be issued tightly due to conflict with patients because a private insurer would refuse subscription of subjects with BAVM. Finally, to secure more accurate diagnoses of BAVM in this cohort, we excluded subjects diagnosed with BAVM who had not undergone any contrast-enhanced CT, MRI, or DSA examination within 2 weeks before or after the diagnosis. Therefore, the estimated number of subjects with BAVM is likely not higher than real statistics.

On the other hand, we reported a mortality rate of $21.1 \%$ for RBAVM patients during the follow-up period. The death information provided by NHIS-NSC included any kind of death that could not be limited by RBAVM. Thus, this mortality rate was an overall mortality rate rather than an RBAVM-related mortality rate, which could be overestimated. In the same context, the rate of severe disability could be overestimated, too.

Although it was not the main theme of this work, we found 140 subjects with BAVM (Q282) diagnostic codes without any hemorrhagic stroke code (I60x, I61x, and I62x), which could be thought to be UBAVM. The standardized incidence was 1.6 per 100,000 person-years. However, we could not estimate the true incidence of UBAVM because the proportion of undetected asymptomatic subjects was not clear. Nevertheless, it is notable that the annual rupture rate of UBAVM was 2.3\% during 661 person-years of observation for 140 subjects. Previous reports regarding the natural history of BAVM have revealed an annual risk of rupture of $2.0-4.2 \% .^{8,12,18,23}$ Although it might be a partial detection rate among undetected BAVM, our estimation of the annual risk of detected UBAVM seems to be consistent with previous findings.

The major limitation of this cohort study is the lack of diagnostic imaging data. The natural history of BAVM largely depends on the location and size of the nidus, as well as on the composition of the draining system. However, the NHIS-NSC does not include imaging data for analyzing morphological classifications. Moreover, because the identification numbers of the patients were anonymized, the subjects could not be matched. Therefore, further investigation is warranted to construct a nationwide registry for BAVM with an appropriate imaging archive to clarify the risk factors for hemorrhage and the efficacy of different treatments. The other limitation is related to inaccuracies of claim data. However, previous studies have validated ICD-10-based definitions for cerebrovascular disease and acute myocardial infarction, which were compared with medical records reviews. The positive predictive values were $83.4 \%$ for ischemic stroke and $>70 \%$ for myocardial infarction (I21), respectively. ${ }^{16,19}$ Thus, the deduced incidence of RBAVM of this investigation should be interpreted in consideration of these limitations.

\section{Acknowledgments}

This study used NHIS-NSC data (NHIS-2016-2-270) created by the NHIS.

\section{References}

1. Al-Shahi R, Bhattacharya JJ, Currie DG, Papanastassiou V, Ritchie V, Roberts RC, et al: Prospective, population-based detection of intracranial vascular malformations in adults: the Scottish Intracranial Vascular Malformation Study (SIVMS). Stroke 34:1163-1169, 2003

2. Al-Shahi R, Fang JS, Lewis SC, Warlow CP: Prevalence of adults with brain arteriovenous malformations: a community based study in Scotland using capture-recapture analysis. J Neurol Neurosurg Psychiatry 73:547-551, 2002

3. Al-Shahi R, Warlow C: A systematic review of the frequency and prognosis of arteriovenous malformations of the brain in adults. Brain 124:1900-1926, 2001

4. Berman MF, Sciacca RR, Pile-Spellman J, Stapf C, Connolly ES Jr, Mohr JP, et al: The epidemiology of brain arteriovenous malformations. Neurosurgery 47:389-397, 2000

5. Brown RD Jr, Flemming KD, Meyer FB, Cloft HJ, Pollock BE, Link ML: Natural history, evaluation, and management of intracranial vascular malformations. Mayo Clin Proc 80:269-281, 2005

6. Brown RD Jr, Wiebers DO, Forbes G, O'Fallon WM, Piepgras DG, Marsh WR, et al: The natural history of unruptured intracranial arteriovenous malformations. J Neurosurg 68:352-357, 1988

7. Brown RD Jr, Wiebers DO, Torner JC, O'Fallon WM: Incidence and prevalence of intracranial vascular malformations in Olmsted County, Minnesota, 1965 to 1992 . Neurology 46:949-952, 1996

8. Crawford PM, West CR, Chadwick DW, Shaw MD: Arteriovenous malformations of the brain: natural history in unoperated patients. J Neurol Neurosurg Psychiatry 49:1-10, 1986

9. Fleetwood IG, Marcellus ML, Levy RP, Marks MP, Steinberg GK: Deep arteriovenous malformations of the basal ganglia and thalamus: natural history. J Neurosurg 98:747750, 2003

10. Gabriel RA, Kim H, Sidney S, McCulloch CE, Singh V, Johnston SC, et al: Ten-year detection rate of brain arteriovenous malformations in a large, multiethnic, defined population. Stroke 41:21-26, 2010

11. Hillman J: Population-based analysis of arteriovenous malformation treatment. J Neurosurg 95:633-637, 2001

12. Itoyama Y, Uemura S, Ushio Y, Kuratsu J, Nonaka N, Wada $\mathrm{H}$, et al: Natural course of unoperated intracranial arteriovenous malformations: study of 50 cases. J Neurosurg 71:805-809, 1989 
13. Jessurun GA, Kamphuis DJ, van der Zande FH, Nossent JC: Cerebral arteriovenous malformations in The Netherlands Antilles. High prevalence of hereditary hemorrhagic telangiectasia-related single and multiple cerebral arteriovenous malformations. Clin Neurol Neurosurg 95:193-198, 1993

14. Kim T, Lee H, Ahn S, Kwon OK, Bang JS, Hwang G, et al: Incidence and risk factors of intracranial aneurysm: a national cohort study in Korea. Int J Stroke 11:917-927, 2016

15. Kim T, Lee H, Bang JS, Kwon OK, Hwang G, Oh CW: Epidemiology of moyamoya disease in Korea: based on national health insurance service data. J Korean Neurosurg Soc 57:390-395, 2015

16. Kimm H, Yun JE, Lee SH, Jang Y, Jee SH: Validity of the diagnosis of acute myocardial infarction in Korean national medical health insurance claims data: the Korean Heart Study (1). Korean Circ J 42:10-15, 2012

17. Lee J, Lee JS, Park SH, Shin SA, Kim K: Cohort profile: the National Health Insurance Service-National Sample Cohort (NHIS-NSC), South Korea. Int J Epidemiol 46:e15, 2017

18. Ondra SL, Troupp H, George ED, Schwab K: The natural history of symptomatic arteriovenous malformations of the brain: a 24-year follow-up assessment. J Neurosurg 73:387391, 1990

19. Park JK, Kim KS, Kim CB, Lee TY, Lee KS, Lee DH, et al: The accuracy of ICD codes for cerebrovascular diseases in medical insurance claims. Korean J Preventive Med 33:7682,2000

20. Stapf C, Mast H, Sciacca RR, Berenstein A, Nelson PK,
Gobin YP, et al: The New York Islands AVM Study: design, study progress, and initial results. Stroke 34:e29-e33, 2003

21. Toffol GJ, Biller J, Adams HP Jr: Nontraumatic intracerebral hemorrhage in young adults. Arch Neurol 44:483-485, 1987

22. Winn HR (ed): Youmans Neurological Surgery, ed 6. Philadelphia: Elsevier, 2011

23. Yamane F, Takeshita M, Izawa M, Kagawa M, Sato K, Takakura K: Natural history of arteriovenous malformations: analysis of non-radically treated patients. J Clin Neurosci 5 Suppl:26-29, 1998

\section{Disclosures}

The authors report no conflict of interest concerning the materials or methods used in this study or the findings specified in this paper.

\section{Author Contributions}

Conception and design: T Kim. Acquisition of data: T Kim. Drafting the article: T Kim. Critically revising the article: Oh, Kwon, JE Kim, Kang, Cho. Statistical analysis: T Kim, Bang, Lee. Study supervision: Oh.

\section{Correspondence}

Chang Wan Oh: Seoul National University Bundang Hospital, Gyeonggi-do, Seoul, Republic of Korea.wanoh@snu.ac.kr. 